\title{
Effect of low energy high fibre and grass silage feeding strategies on metabolic status of dairy cows in the peri-partum period
}

\section{Butler ${ }^{1,2}$, J Patton $^{1}$, J J Murphy ${ }^{1}$, F J Mulligan $^{2}$}

${ }^{1}$ Dairy Production Research Centre,Teagasc, Moorepark, Fermoy, Co. Cork, Ireland, ${ }^{2}$ School of Agriculture, Food Science and Veterinary Medicine, University College Dublin, Dublin 4, Ireland

Email: michael.butler@teagasc.ie

Introduction The dry period is an important phase of the dairy cow's lactation cycle. Plane of nutrition during this time can have a major influence on peripartum metabolism (Grummer, 1995). Many studies have focused on developing feeding strategies for the transition period ( $3 \mathrm{wks}$ pre-partum to $3 \mathrm{wks}$ post-partum) that alleviate the extent of metabolic stress. It has been shown recently that feeding a low energy, high-fibre total mixed ration (TMR) for the entire dry period may be beneficial in terms of improving metabolic status and reducing incidence of peripartum health disorders (Dann et al., 2006). The aim of this study was to compare the metabolic profiles of Holstein Friesian cows managed under a high fibre TMR dry cow regime, relative to a moderate quality grass silage diet.

Materials and methods Sixty mature spring-calving Holstein-Friesian cows were blocked according to expected calving date, genetic merit for milk yield, body condition score (BCS), bodyweight and parity. Cows were randomly assigned to one of the two dry period nutritional treatments at drying off: (1) high fibre TMR (HF-TMR) or (2) grass silage (GS). The HF-TMR diet consisted of 0.40 wheaten straw, 0.25 grass silage, 0.25 maize silage and 0.10 soyabean meal. Both diets were offered ad libitum for the duration of dry period, and were supplemented with an appropriate dry cow mineral mix. Net energy density was calculated as 0.71 UFL (Unité Fourragère Lait) and 0.78 UFL for the HF-TMR and GS diets, respectively. Blood samples were taken from cows by coccygeal venipuncture, for week 1 pre-partum to week 4 postpartum. Samples were collected via evacuated vials containing, lithium heparin as an anticoagulant. Plasma analyte profiles were analysed using appropriate kits each containing a number of reagents for each analyte on an ABX Mira Auto analyser. Plasma insulin-like growth factor I (IGF-I) concentrations were determined by a validated double-antibody radioimmunoassay after ethanol-acetone-acetic acid (60:30:10) extraction. Plasma insulin concentrations were determined by using a solid-phase fluoroimmunoassay. Repeated measures analysis of treatment effects on plasma metabolites, insulin and IGF-I was carried out using the MIXED procedure of SAS (SAS, 2006). A first order autoregressive covariance structure was used. Treatment, time and treatment by time interactions were tested. Cow was included as a random effect nested within treatment. Plasma analyte data were not normally distributed and were therefore log-transformed prior to statistical analysis. Plasma analyte data are presented in as means of log-transformed values for wk 1 pre-partum to wk 4 post-partum.

Results Plasma metabolite profiles were similar for both dry cow treatments during wk 1 pre-partum to wk 4 post-partum, except for a greater mean betahydroxybutyrate (BHB) concentration for treatment GS (Table 1). Plasma calcium concentration tended to be greater for HF-TMR compared to GS during this time. Liver enzyme GLDH (Glutamatedehydrogenase) was greater for treatment HF-TMR relative to treatment GS Dry period diet did not affect peripartum concentrations of insulin or IGF-I .

Table 1 Effect of dry period diet on plasma analyte concentrations

\begin{tabular}{lllll}
\hline \hline & HF-TMR $^{1}$ & $\mathrm{GS}^{1}$ & s.e.d. & P-value \\
\hline Plasma NEFA, mmol/1 & $-0.34^{2}$ & -0.37 & 0.085 & 0.66 \\
Plasma BHB, mmol/1 & -0.12 & -0.31 & 0.078 & 0.02 \\
Plasma triglyceride, mmol/1 & -1.83 & -1.82 & 0.035 & 0.81 \\
Cholesterol, mmol/1 & 0.91 & 0.90 & 0.053 & 0.87 \\
Magnesium, mmol/1 & -0.15 & -0.18 & 0.028 & 0.28 \\
Phosphorus, mmol/l & 0.43 & 0.41 & 0.055 & 0.73 \\
Calcium, mmol/l & 0.84 & 0.82 & 0.012 & 0.11 \\
Aspartate-aminotransferase, iu/l & 3.79 & 3.75 & 0.045 & 0.34 \\
GLDH, iul/l & 2.63 & 2.33 & 0.144 & 0.03 \\
Bilirubin, $\mu \mathrm{mol} / \mathrm{l}$ & 1.97 & 2.04 & 0.061 & 0.23 \\
Insulin, $\mu \mathrm{IU} / \mathrm{ml}$ & 1.31 & 1.30 & 0.080 & 0.85 \\
IGF-I, ng/ml & 4.63 & 4.57 & 0.090 & 0.52 \\
\hline \hline
\end{tabular}

${ }^{1}$ HF-TMR = High-fibre Total Mixed Ration; GS = Grass silage ${ }^{2}$ All data in table log-transformed .

Conclusions Effects of dry period diet on plasma analyte profiles during the peripartum period were modest overall. Differences in plasma BHB concentration are consistent with greater body tissue mobilisation for GS, arising from a higher level of body lipid accretion during the dry period. Evidence from this study of a beneficial effect of HF-TMR on peripartum Ca metabolism is tenuous. The reasons for elevated GLDH for HF-TMR compared to GS are somewhat unclear; however neither treatment had GLDH concentrations that would be indicative of compromised liver function.

\section{References}

Dann, H.M., Litherland, N.B., Underwood, J.P., Bionaz, M., D`Angelo, A., Mc Fadden, J.W. and Drackley, J.K. 2006. Journal of Dairy Science 89, 3562-3577.

Grummer, R.R. 1995. Journal of Animal Science 73, 2820-2833.

SAS ${ }^{\circledR}$ User's Guide 2006. Version 8.2.0 Edition. SAS Institute., Canada, USA. 